\title{
"Chemsex" and harm reduction need among gay men in South London
}

Bourne A $(\mathrm{PhD}, \mathrm{BSc})^{1 *}$, Reid D $(\mathrm{MSc}, \mathrm{BSc})^{1}$, Hickson F (PhD, BSc, BA $)^{1}$, Torres-Rueda S (MSc, BA) ${ }^{2}$, Steinberg $\mathrm{P}(\mathrm{BA}, \mathrm{MA})^{3}$, Weatherburn $\mathrm{P}(\mathrm{MSc}, \mathrm{BA})^{1}$

1. Sigma Research, Department of Social \& Environmental Health Research, London School of Hygiene \& Tropical Medicine.

2. Department of Global Health and Development, London School of Hygiene \& Tropical Medicine.

3. Public Health, London Borough of Lambeth

${ }^{*}$ Corresponding author:

Dr Adam Bourne

Sigma Research

London School of Hygiene \& Tropical Medicine

15-17 Tavistock Place

London WC1H 9SH

Email: adam.bourne@Ishtm.ac.uk

Tel: 02079272793

Word count: 4655

This is the final version of the paper submitted by the authors to the International Journal of Drug Policy for design and print production (June 29, 2015).

The final printed edition is available at http://www.sciencedirect.com/science/article/pii/S0955395915002145

doi:10.1016/i.drugpo.2015.07.013 


\section{"Chemsex" and harm reduction need among gay men in South London}

\section{Background}

Chemsex is a colloquial term used by gay men in some parts of the UK to describe the use of psychoactive substances (typically mephedrone, GHB/GBL or crystal methamphetamine) during sex. Use of these drugs by gay men in London appears to have risen sharply from relatively low levels and, as yet, there is little data to inform appropriate harm reduction services. This study sought to understand the personal and social context of chemsex and the nature of harm reduction need.

\section{Methods}

In-depth interviews were conducted with thirty self-identifying gay men (age range 21-53) who lived in three South London boroughs, and who had used either crystal methamphetamine, mephedrone or GHB/GBL either immediately before or during sex with another man during the previous 12 months. Data were subjected to a thematic analysis.

\section{Results}

While around half of participants had utilised a range of drugs over many years, others had only recently been introduced to drugs, often by sexual partners who wished to enhance the sexual session. As relatively new drugs on the gay scene, understanding of appropriate dosing was lacking and a majority described overdoses, particularly in relation to GHB/GBL. Negotiation of sex, especially in group sex environments, was complicated by the effects of the drugs and a small number of men reported concerns relating to sexual consent. While a significant proportion of men had experienced a range of physical and mental health harms, few had accessed professional support for fear of judgement or concern about chemsex expertise.

\section{Conclusion}

Findings from this study indicate a substantial degree of harm in the usage of relatively new psychoactive substances in highly sexual circumstances. Generic drug services, typically designed to address the needs of opiate users, may not be sufficiently resourced to address the specific and acute needs of gay men engaging in chemsex.

Key words: chemsex; MSM; crystal methamphetamine; GHB/GBL; sexual consent 


\section{Introduction}

Harms arising among gay, bisexual and other men who have sex with men (MSM) who use drugs are both general and specific. They are general in that drug use among MSM is particularly high (Crime Survey of England \& Wales, 2014 (CSEW)) and they are therefore disproportionately subject to the harms usually associated with illicit drugs, including psychological difficulties and adverse health outcomes. MSM who use drugs are also subject to specific harms arising from their use in environments or cultural contexts particular to gay men and other MSM. This paper explores harms and harm reduction need among self-identified gay men who use mephedrone, gammahydroxybutrate/gamma-butralactone (GBL/GBL) or crystal methamphetamine during sex - a behaviour commonly referred to in the UK as "chemsex".

Gay and bisexual men have higher rates of substance use than age-comparable heterosexual men. Data from the 2013 Crime Survey of England \& Wales indicate that gay and bisexual men were three times as likely to have used an illegal drug within the previous year (33\% of respondents) compared to heterosexual people (11\%). Reasons for higher rates of both drugs and alcohol among gay men (see Bourne., 2012 for review of global evidence) require further exploration, but most theories point to the effects of minority stress (Mayer., 1995) or highlight how gay social activity is predominantly associated with bars and clubs where alcohol is served and where drugs are both accessible (as demonstrated by an 'in situ' survey of MSM in London gay commercial venues, Measham, Wood, Dargan \& Moore., 2011) and normalised (Keogh, Reid, Bourne, Hickson \& Weatherburn., 2009). In particular, gay men in London are viewed as 'early adopters' of drug trends for the rest of the drug using population (Measham et al., 2011).

While use of opiates or crack cocaine among MSM have historically been very low, use of ecstasy, cocaine and MDMA (3,4-methylenedioxy-methamphetamine) have been popular among gay communities in the UK for many years (Keogh et al, 2009). Since 2011 there has been an increasing amount of survey, clinic and practitioner evidence suggesting a rise in new psychoactive substances, both within heterosexual and MSM populations (Drugscope., 2014; Stuart., 2013a; Kirby \& ThornberDunwell., 2013), with particular concern focussed on mephedrone and GHB/GBL. The 2010 European MSM Internet Survey (EMIS) recorded that 2.9\% of the 15,423 MSM respondents living in 
England had used mephedrone and $1.6 \% \mathrm{GHB} / \mathrm{GBL}$ within the previous 4 weeks. Use is significantly higher in London, with 5.2\% reporting use of mephedrone, and 5.5\% GHB/GBL (Bourne, Reid, Hickson, Torres Rueda \& Weatherburn., 2014). However, data from the CSEW suggests that use of both of these drugs may have risen in the time since data collection for the EMIS study, with corresponding reductions in the use of ecstasy and cocaine. A number of reports in the gay and mainstream media reinforce this impression, particularly in London (Morrison., 2014). There is also a suggestion that use of crystal methamphetamine, which had remained low and relatively stable among MSM for much of the last decade (Bonell, Hickson, Weatherburn \& Reid., 2010) may also be rising, although perhaps not as rapidly as the other two drugs.

Significant concern has been expressed by health care providers, the gay and mainstream media and social commentators about the impact of drug use on the physical, mental and social well-being of gay men and the broader impact on gay community connectivity (Stuart., 2013b; Zapata., 2013). Within the past two years there have been a number of drug-related casualties among gay men in clubs or sex-on-premises venues in Vauxhall (a major gay commercial area in the northern part of Lambeth, close to the River Thames) that have been reported in the media (Morgan., 2012; Hopkins., 2011; Reid-Smith., 2012), indicating a need for drug harm reduction among this local population. Between 2007 and 2012 there were 96 ambulance call outs relating to accidental or unknown drug overdose in the Vauxhall area during the hours of the night-time economy, although it is not possible to disaggregate this data by sexual orientation (Clark., 2013). The drug most commonly associated with emergency admission to St. Thomas's Hospital in Lambeth in 2010 was GHB/ GBL, resulting in 270 presentations (Wood, Greene \& Dargan., 2013), although similarly it is not possible to disaggregate the data to identify who among them were gay or other MSM.

In addition to the general health hazards of drug use, gay men face issues relating to higher risk sexual behaviour and the possibility of HIV/STI transmission while under the influence of these drugs (Bourne, Reid, Hickson, Torres Rueda \& Weatherburn., submission under review; Vosburgh et al, 2012). Men's reasons for using drugs during sex are variable and complex, and are explored elsewhere (Bourne, Reid, Hickson, Torres Rueda \& Weatherburn., in preparation). GHB/GBL is a nervous system depressant (Brennan and Van Hout., 2014), while mephedrone and crystal 
methamphetamine are stimulants. All three can trigger feelings of euphoria and among many users can also facilitate intense feelings of sexual arousal. This is particularly the case for crystal methamphetamine (Kurtz, 2005).

A significant body of media associates use of these three drugs with sexual activity in a manner that has not previously been documented among this population in the UK (e.g. Daly., 2013; Hopkins., 2014; Taylor., 2014). Typically termed "chemsex", combining sex and drugs has also been described a "Party and Play" (sometimes abbreviated to "PnP") in North America and 'intensive sex partying' in Australia (Hurley and Presage., 2009). Associations between drugs and sex are not new, however sex under the influence of previously popular drugs such as ecstasy and cocaine was often incidental, rather than planned and intentional, as has been reported the case with GHB/GBL, mephedrone or crystal methamphetamine.

Perceived changes in the drugs most popular among gay men in London, mirrored by high profile drug related deaths in gay commercial venues, led us to devise a study to explore the personal and social context of use of these psychoactive substances among MSM in order to better understand how to respond to the phenomenon via a harm reduction approach. There is limited qualitative evidence relating to drug use among this population and, to date, no research from London that explores the situated reality of chemsex as a phenomenon of significant public health concern. Our focus within this paper is was on experience of, or exposure to, harm relating to chemsex and the harm reduction services that might be required to address such issues.

\section{Methods}

In-depth interviews were conducted with 30 men who were resident in three London boroughs (Lambeth, Southwark and Lewisham) that have both large gay populations and a high prevalence of diagnosed HIV (Yin et al., 2014). One of these boroughs (Lambeth) also contains a large, commercial gay scene, including numerous bars, clubs and sex-on-premises venues (SoPVs). To be eligible for the study, men had to be aged 18 or over, be resident in one of the three boroughs and to have used either crystal methamphetamine, mephedrone or GHB/GBL either immediately before or during sex with another men (or men) at least once during the preceding 12 months. 
Participants were recruited via push notifications on a mobile phone based geo-social networking application (app) that caters to gay men, as well as paid advertising in a large, London gay-scene publication and referrals from partner charities that cater to the needs of gay and bisexual men. Promotional business cards in commercial gay premises in Lambeth were also distributed. All promotional materials directed potential participants to a dedicated webpage that provided further details about the project and a means of contacting the researchers to arrange a time for interview. . In total, 44 men initially registered interest, of whom 9 were ineligible to participate given that they were not resident in LSL, and a further 5 did not respond to follow-up emails to arrange a time for interview.

All of the men who ultimately comprised this sample described themselves as gay. Table 1 illustrates the demographic characteristics of participants.

Table 1: Participant demographic characteristics; residency \& HIV testing history

\begin{tabular}{|l|l|l|l|}
\hline HIV testing history & $\mathbf{N}$ & Age & \\
Diagnosed HIV positive & 13 & Mean & Re years \\
Last test negative & 17 & Range & \\
\hline London borough of & $\mathbf{N}$ & Ethnicity & $\mathbf{N}$ \\
residence & 14 & White British & 16 \\
Lambeth & 11 & White Other & 8 \\
Southwark & 5 & White Irish & 3 \\
Lewisham & & Black Caribbean & 1 \\
& & Other & 2 \\
\hline
\end{tabular}

In-depth interviews, lasting one to two hours, explored men's history of drug use, a profile of their current sexual lives, their perceived impact of drugs on sexual behaviour, experiences of harm and perceptions or experiences of accessing services or support (professional or otherwise) in relation to their drug use. Interviews were transcribed verbatim and thematically analysed using an inductive 
approach (Braun \& Clarke., 2006). The data were read and initial codes were documented, organised into relevant themes and all examples of each potential theme were recorded. Identification of key themes was undertaken by one author and corroborated by two others. This research was granted approval by the Research Ethics Committee of the London School of Hygiene \& Tropical Medicine. Quotes, shown in italics, are followed by the age and HIV testing history of the interviewee.

\section{Results}

We begin by briefly outlining participants' drug use history, as well as the frequency of their usage and an illustration of commonplace behaviours during chemsex. This is followed by: consideration of drug dosing issues (including harm associated with overdose); an account of perceived complexities of sexual consent in a chemsex environment; their experience of, or exposure to, acute mental health problems; and their experience or perceptions of managing their drug use, via engagement with support and services, professional or otherwise.

\section{Frequency, history and context of drug use during sex}

In terms of drug taking histories, around half of participants had used a broad range of drugs over their lifetimes, with newer psychoactive substances being the latest and most readily available. Others had only recently began using drugs - and doing so during sex - and had usually been introduced to them by sexual partners.

"I was always anti-drugs, yeah, very much. Basically I used to fancy this guy very, very much. And when he came to my place and said, 'Oh, would you like some?' Because I fancy him I thought, 'Okay. So let's share some together' [...] so the first time we snorted some mephedrone. We smoked some meth. And we had a bit of G, you know, it's the kind of normal concoction of gay sex with them drugs." [Aged 31, last tested HIV negative]

The extent and frequency of drug use during sex varied considerably among the men interviewed. Three men only used chemsex drugs to provide an 'edge' to sex with regular or romantic partners, or only did so with casual partners on an infrequent basis. At the other end of a behavioural spectrum were men who used these drugs very regularly and who engaged in chemsex on a near daily basis. 
Poly-drug use (the use of multiple substances in the same session) was commonplace. Mephedrone was used by all but 3 participants, with two thirds typically doing so in combination with GHB/GBL. Crystal methamphetamine was used on a regular basis (i.e. at least weekly) by a third of our sample, all of whom typically used GHB/GBL within the same session.

Chemsex typically occurred in private homes, and was also commonly reported in commercial sex-onpremises venues (SoPVs) such as saunas or sex clubs. While one-on-one sex (often with romantic partners) was reported, the chemsex that participants engaged in most commonly occurred as a group sexual activity. Men had sex with numerous other men, serially (one after another over a prolonged session). A majority of participants described an increased propensity to engage in more adventurous sexual activity while under the influence of drugs (especially the case with crystal methamphetamine) including ano-brachial intercourse ('fisting'), and being anally receptive to multiple men in quick succession as part of a group session or orgy.

\section{Modes of drug delivery and associated harm}

Both mephedrone and crystal methamphetamine can be snorted and this was the preferred means of administering these drugs for the majority who regularly used them. However, a third of participants had injected either one or the other (a behaviour commonly referred to as 'slamming'), almost all of whom had done so in the previous year. The terminology 'slamming' appeared to represent a desire to distinguish, or distance, oneself from opiate drug users who were negatively perceived. In every instance of injection drug use described, participants clearly stated that careful use was made of clean needles and that they endeavoured to adhere to safe injection practices. It was very common for men to be injected by others who they believed had experience of how to inject safely.

And I know someone who's in the business of Tina [crystal methamphetamine] who will go to people who are too frightened of slamming themselves. He will visit their house, slam them and leave. Visit their house, slam them and leave. Like a nurse drug dealer. [Aged 48, diagnosed HIV positive] 
Several men did, however report that they were aware of other men and other chemsex parties where the use of clean needles was not so strictly observed. Seven men reported issues arising from injection site injuries, including collapsed veins and muscle damage. Four men described intra-rectal needleless insertion of mephedrone, a behaviour often referred to as 'booty bumping'. Two of those reporting such behaviour described acute damage, including rectal fissures.

\section{Overdosing and associated harm}

The most acute and immediate harm arising from chemsex related to overdosing, particularly of GHB/GBL. This drug is typically administered orally, diluted in very small quantities (generally between 0.5 and $1 \mathrm{ml}$ ) in a soft drink, the consumption of which has to be timed in order to avoid overdose. However, control of dosing can also become more difficult as the effect of the drug accumulates over time, particularly when it is often taken in combination with mephedrone and/or crystal methamphetamine, or alongside alcohol. An overdose of GHB/GBL can result in a state of unconsciousness often referred to as a G-hole. The majority of men who reported use in the last year had overdosed on one or more occasions.

"I need somebody else to tell me you are a bit fucked, go and have a lie down. Because it's one of those things where it is kind of slow acting and it will cumulatively dope up in your system. You don't know that you have gone over the edge until you are falling off of the cliff face." [Aged 50, last tested negative]

Short bouts of a loss of consciousness were very common but were perceived as relatively normal during sex on GHB/GBL. Some men described regaining consciousness only to find that significant periods of time had passed, they had lost control of their bowels or bladder, were in pain or vomiting. Participants commonly reported seeing, or having heard of, other gay men who had been hospitalised or who had died as a result of GHB/GBL overdosing - typically resulting from respiratory depression or choking while unconscious. Five participants had been hospitalised due to severe overdose and described negative experiences of censorious medical staff, in addition to their own sense of shame or horror at their actions. 
Three men described regaining consciousness to find that they were being anally penetrated, or suspected they had been while unconscious. In each instance, the men concerned had overdosed on GHB/GBL, perhaps in combination with other drugs or alcohol. The sex that occurred in such circumstances was, in all cases, non-consensual. However, the men were reluctant to refer to this as sexual assault or rape, although some still found the experience distressing. Many participants articulated what they considered to be a 'blurry line' regarding consent during chemsex. Overdosing meant that they, or their sexual partners, might drift in and out of consciousness or may cycle between pleasure and distress while having sex. In the following quote a participant describes an incident he witnessed at a private sex party where he felt consent was difficult to establish.

"I went to party and there was only one person there who was passive [the receptive anal intercourse partner]; everyone else was active [the insertive anal intercourse partner]. And I felt uncomfortable because the person who was passive was rolling around in bed moaning and sweating, 'Oh l've done too much G', and he was in a real state. I didn't feel comfortable having sex with him because I didn't feel it would be consensual, but everyone else had no problem scrabbling around on top of him." [Aged 21, last tested HIV negative]

Beyond concerns relating to GHB/GBL overdosing, five participants described acute irritability, anxiety or aggression relating to an overdose of crystal meth. In some instances men required medical interventions for extreme paranoia and anxiety attacks following particularly intense chemsex sessions. Among around a sixth of our sample , chemsex was blamed for longer term harms to their psychological well-being, including depression, anxiety and psychosis.

\section{Harms to social and relational well-being}

Two-thirds of participants lamented the inebriated and often mechanical interaction with other men during chemsex, which stood in stark contrast to other social relationships or their ideal romantic relationship. Drug use was sometimes seen as reducing consideration for others and promoting sexual selfishness. 
"I think it just makes you really selfish. I think that crystal makes you selfish so if you're a bottom you really want to get fucked. 'My turn now, my turn in the sling, fist me.' Whoever the guy is. I see this. Makes you selfish. It's about me, it's about me." [Aged 48 years, diagnosed HIV positive]

Some also described damage to relationships or hurt caused to partners, friends and families as a result of prioritising chemsex over social engagements or other obligations. Nearly half of men discussed the effect of chemsex on their employment, ability to work effectively and career development. Most commonly they reported missing work because of withdrawal, poor concentration and diminished cognitive ability, which affected their performance detrimentally.

\section{Personal reflections and responses to harm}

Participants broadly fell into three categories of exposure to and/or recognition of harm: those who used drugs (either regularly or infrequently) and who felt in control of their use and managed their dosing, frequency and dependency carefully and effectively; those who felt that their drug use was problematic and having a deleterious effect on their physical, mental or social well-being and had sought - or were actively considering seeking - professional help to manage or reduce their drugs use; and finally those who, objectively speaking (from the perspective of the interviewers), may have had a problematic relationship with drug use during sex but had not recognised or responded to this. The men in this latter group were often exposed to, or experiencing, a significant degree of sexual, mental or physical health related harm but had been unable or unwilling to control or manage their drug use more effectively. Maintenance of chemsex behaviour was often facilitated by favourable comparison to other men who engaged more frequently, or who used drugs or drug delivery methods that they perceived as more problematic. For example, those men that did not take crystal methamphetamine considered it a much more dangerous drug than those who were taking it. Among crystal methamphetamine users, those who did not inject held only this delivery mechanism as the problem behaviour. Even among those that injected, some described this act as relatively safe because, unlike others they heard of, they were not sharing needles and not using heroin. 
Those men who recognised when their drug use was problematic, or had the potential to become so, employed a number of strategies to manage or control their use. Some tried to limit themselves to having chemsex on only certain days of the week or at certain times of the year. Others limited the volume of drugs used by carrying a very limited quantity when they planned a chemsex session. Some tried to avoid certain drugs (particularly crystal methamphetamine), or social settings or friends who they associated with chemsex.

"I have to stop going on the gay scene properly because I will take drugs and I will just go from one club to another to another so I have to limit my clubbing now. I used to go clubbing like four days a week and have a chill-out for two days and I would have a nap in between. I couldn't do it anymore. I am getting older and I am starting to feel older and I don't want to... end up in a box too early." [Aged 36 years, diagnosed HIV positive]

\section{Engagement with, or perceptions of, professional services}

Half of participants had never accessed any kind of professional support and those who had usually only accessed drugs information websites. A small number had accessed psychotherapeutic services, usually in community-based settings, and they valued the opportunity to talk through options to better manage their engagement in chemsex, rather than being told they had to abstain. Regardless of the mechanism, men valued clear, honest and non-judgemental advice about engaging in chemsex and how to manage potential harms.

Most participants had not sought out specialist drug services because they were concerned that they might be ill-equipped to discuss chemsex among gay men, and its associated context and costs and benefits. Some had concerns about disclosing their sexuality or, more commonly their sexual activity under the influence of drugs to others who may have limited cultural understanding. When asked to consider where they would feel most comfortable accessing drug information or harm reduction services relating to chemsex, the vast majority said they would prefer to visit a sexual health service. They had come to know and trust these organisations as gay friendly and staffed by individuals aware of the social and cultural context of gay sex. 
Finally, more than a quarter of participants expressed a desire to see more combined drug harm reduction and sexual health services, where specialist skills could address the holistic health needs of gay men.

"There are a lot of services for sexual health and then there are a couple of services offering for substance use and I definitely think there needs to be a lot more combined services. There are a lot of psychological factors driving both and they are very much interlinked [...] I think the underlying issues are a lot more prominent than people believe or people acknowledge or people understand in terms of what is driving the drug use in the gay scene." [Aged 24, last tested HIV negative]

\section{Discussion}

This paper reports findings from a qualitative investigation of a newly emerging and fast developing phenomenon in London, UK. Drug use has always been more common place among gay and bisexual men, than the wider adult male population, but the drugs most commonly used and the situations within which use occurs have changed significantly, warranting reflection on, and revision of, harm reduction services to meet extant need. Men were only recruited from three South London Boroughs (a relatively confined geographical area) but, given the rapid transit of gay men across the city and the immediacy of smartphone sexual networking applications, it is likely that these findings may reflect the needs of men residing in other parts of London and in other large cities - especially those with larger gay male populations where the drugs of choice are similar to those explored in this study.

This paper highlights acute harm reduction need among a population that has not typically been in receipt of drug interventions. Some authors have argued that the fact that drug use among gay men has not commonly been associated with other criminal or antisocial activity (as is sometimes the case with use of opiates [Keogh et al., 2009]) has meant their needs have been overlooked. That three out of 30 men who took part had been the victim of non-consensual sex (and several others mentioned they had witnessed sex where the issue of consent-giving seemed problematic) is concerning. Many participants felt that the notion of consent was conceptually complicated in a (group) chemsex 
encounter where the majority of those present are highly sexually charged, and where some describe a difficulty controlling their sexual desire (Bourne et al., submission under review). This situation requires careful consideration but, at the very least, interventions should be designed to encourage men to be attentive to the cognitive capacity of their sexual partners and to ascertain whether sex is consensual. Those men who feel they have been the victim of non-consensual sex should be made aware of support mechanisms and linked to post-exposure prophylaxis for HIV where appropriate.

Many of the harms experienced stem from a lack of understanding of how to use these drugs safely. There has been a rapid transition from the use of drugs such as ecstasy and cocaine that had been popular on the gay scene for more than a decade towards these new psychoactive substances. While definitive data is yet to be published, use of mephedrone, GHB/GBL and, perhaps to a lesser extent, crystal methamphetamine appear to have risen in a short period suddenly. The speed of this transition may mean that the shared community understanding around safe drug use (including dosing, combinations, safe injection practices and dealing with undesired effects) that have existed for previously popular drugs is still developing in relation to these newer substances. There is a clear need for factual, honest and non-judgemental information about safe use of these drugs to be promoted to men currently engaging in chemsex, or those considering doing so in the future. Existing good practice in this area should be identified and scaled-up where resources are available.

Over the last 10 years there has been significant investment in sexual health services for gay men, to ensure they are accessible, acceptable and comfortable environments in which they can talk honestly about their sexual behaviour without fear of judgement. Among EMIS survey respondents resident in England, $96.4 \%$ of MSM said they felt they had been treated with respect the last time they tested for HIV (The EMIS Network., 2011). It is, therefore, unsurprising that when faced with managing a personally sensitive issue, men would prefer to discuss their drug use in a sexual health setting. However, meaningful response to the harms associated with chemsex could place significant strain upon the material resources of sexual health clinics that are not currently commissioned to deliver drug harm reduction services. While there are emerging examples of good practice in terms of integrating sexual health and drug harm reduction work in London (see, for example, 56 Dean Street and The Burrell Street Clinic services), many sexual health clinics lack the training or capacity to 
respond in depth to drug use in sexual settings among their gay male clients. Local authorities, particularly those with large populations of gay and bisexual men, need to consider joint commissioning structures across public health areas for gay men engaging in chemsex so that the acute harm reduction needs of this population do not fall between the gaps of current commissioning practice.

Injection of drugs among gay men in the UK and other developed countries has historically been very low (for review see Bourne., 2012). This likely reflects the fact that there was limited possibility or additional benefit to the injection of previously popular drugs such as ecstasy and cocaine as opposed to oral or nasal administration. This stands in contrast to the fact that in a convenience, qualitative sample of gay men, a third had injected recently. It is encouraging to note, however, that none of the men interviewed in this study reported the sharing of needles between sexual partners. Most were broadly aware of the potential risks from doing so, but there was still evidence of safe injecting information provision need given that several men reported problems with injection site injuries. However, our finding stands in stark contrast to other research and to media reporting of 'slamming' where there is a perception that safe injection practice is not the norm (Channel 4 News., 30 January 2014) and data reported from gay drugs charity, London Friend, that 70\% of their clients in 2012 had shared needles (Stuart., 2013a). The difference in findings may be the result of a social desirability bias on the part of those we interviewed, or may simply suggest that many sections of the gay communities engaged in chemsex are familiar with safe injection advice. This issue in particular requires further exploration.

Research interest in drug use among gay men has typically focussed on the extent to which use might influence HIV or other STI transmission risk behaviour (e.g. Forrest et al., 2010; Carey et al., 2009). Far fewer studies have explored the role of drugs in gay men's lives or have explored the harms gay men experience when using drugs that extend beyond sexual health. Findings from this study indicate a substantial degree of harm in the usage of relatively new psychoactive substances. Further research is required to determine: the extent of chemsex within the gay male population; the extent of harm experienced; the best means of supporting gay men to better manage or control their drug use where they feel they need to (including examples of existing good practice); and what 
motivates men to use drugs in sex, namely what they value about chemsex and the role that drugs play in their sexual lives.

\section{Acknowledgements}

This research was commissioned and funded by the London Boroughs of Lambeth, Southwark and Lewisham. We also thank all the community-based organisations who helped recruit to, or host, the interviews conducted.

\section{References}

Bonell, C. P., Hickson, F. C., Weatherburn, P., \& Reid, D. S. (2010). Methamphetamine use among gay men across the UK. International Journal of Drug Policy, 21(3), 244-246. doi:

10.1016/j.drugpo.2009.07.002.

Bourne, A. (2012). Drug use among men who have sex with men: Implications for harm reduction. In C. Stoicescu (Ed.), Global State of Harm Reduction 2012 (pp. 147-155). London: Harm Reduction International.

Bourne, A., Reid, D., Hickson, F., TorresRueda, S., \& Weatherburn, P. (2014). The Chemsex study: drug use in sexual settings among gay and bisexual men in Lambeth, Southwark and Lewisham. London: Sigma Research, London School of Hygiene \& Tropical Medicine.

Bourne, A., Reid, D., Hickson, F., TorresRueda, S., \& Weatherburn, P. (in preparation). Meanings and motivations associated with drug use in sexual settings ("chemsex") among gay men in South London.

Bourne, A., Reid, D., Hickson, F., TorresRueda, S., \& Weatherburn, P. (submission under review). Illicit drug use in sexual settings ("Chemsex") and HIV/STI transmission risk among gay men in South London: findings from a qualitative study. 
Braun, V., \& Clarke, V. (2006). Using thematic analysis in psychology. Qualitative Research in Psychology, 3, 77-101.

Brennan, R., \& Van Hout, M. C. (2014). Gamma-hydroxybutyrate (GHB): a scoping review of pharmacology, toxicology, motives for use, and user groups. Journal of Psychoactive Drugs, 46(3), 243-251. doi: 10.1080/02791072.2014.921746.

Carey, J. W., Mejia, R., Bingham, T., Ciesielski, C., Gelaude, D., Herbst, J. H., . . Stall, R. (2009). Drug use, high-risk sex behaviors, and increased risk for recent HIV infection among men who have sex with men in Chicago and Los Angeles. AIDS \& Behavior, 13(6), 1084-1096. doi: 10.1007/s10461008-9403-3.

Clark, C. (2013). A health needs assessment for men who have sex with men (MSM) in Lambeth London: Lambeth Clinical Commissioning Group.

Daly, M. (2013, 3rd June 2013). The Meth-Fuelled, Week-Long Orgies Ravaging London's Gay Sex Party Scene. Vice.

Drugscope. (2014). Business as usual? A status report on new psychoactive substances (NPS) and 'club drugs' in the UK. London: Drugscope.

Forrest, D. W., Metsch, L. R., LaLota, M., Cardenas, G., Beck, D. W., \& Jeanty, Y. (2010). Crystal methamphetamine use and sexual risk behaviors among HIV-positive and HIV-negative men who have sex with men in South Florida. Journal of Urban Health, 87(3), 480-485. doi: 10.1007/s11524009-9422-z.

Hopkins, A. (2011, 22nd December 2011). Clubbed to death? Time Out.

Hopkins, A. (2014, 18th February 2014). Chemsex revealed: Part 1. Beige Magazine. 
Hurley, M., \& Prestage, G. (2009). Intensive sex partying amongst gay men in Sydney. Culture, Health \& Sexuality, 11(6), 597-610. doi: 10.1080/13691050902721853.

Keogh, P., Reid, D., Bourne, A., Weatherburn, P., Hickson, F., Jessup, K., \& Hammond, G. (2009). Wasted opportunities: problematic alcohol and drug use among gay men and bisexual men. London: Sigma Research.

Kurtz, S. P. (2005). Post-circuit blues: motivations and consequences of crystal meth use among gay men in Miami. AIDS \& Behavior, 9(1), 63-72. doi: 10.1007/s10461-005-1682-3.

Mayer, I. H. (1995). Minority stress and mental health in gay men. Journal of Health \& Social Behaviour, 36(1), 38-49.

Measham, F., Wood, D., Dargan, P., \& Moore, K. (2011). The Rise in Legal Highs: Prevalence and patterns in the use of illegal drugs and first and second generation 'legal highs' in south London gay dance clubs. Journal of Substance Use, 16(4), 263-272.

Morgan, J. (2012, 28th October 2012). Man dies at London gay sauna, Gay Star News.

Reid-Smith, T. (2012, 11th July 2012). Man found dead at Chariots, London's biggest gay sauna, Gay Star News.

Office for National Statistics (2014). Drug misuse: Findings from the 2013/14 crime survey for England and Wales. London: Office for National Statistics.

Stuart, D. (2013a, 22nd January 2013). Is London's gay scene self-harming through sex and drug use? Pink News. Retrieved from http://www.pinknews.co.uk/2013/01/22/comment-is-londons-gayscene-self-harming-through-sex-and-drug-use/. 
Stuart, D. (2013b). Sexualised drug use by MSM: background, current status and response. HIV Nursing, 13(1), 6-10.

Taylor, S. (2014, 10th April 2014). Slings, Slamming and Chem-sex, The Huffington Post. Retrieved from http://www.huffingtonpost.co.uk/dr-steve-taylor/hepatitis-c- b 4750791.html

The EMIS Network. (2013). The European Men-Who-Have-Sex-With-Men Internet Survey. Findings from 28 countries. Stockholm: European Centre for Disease Prevention and Control.

Vosburgh, H. W., Mansergh, G., Sullivan, P. S., Purcell, D. W. (2012). A review of the literature on event-level substance use and sexual risk behavior among men who have sex with men. AIDS and Behavior, 16, 1394-1410.

Wood, D. M., Greene, S. L., \& Dargan, P. I. (2013). Five-year trends in self-reported recreational drugs associated with presentation to a UK emergency department with suspected drug-related toxicity. European Journal of Emergency Medicine, 20(4), 263-267. doi:

10.1097/MEJ.0b013e3283573115.

Yin, Z., Brown, A. E., Hughes, G., Nardone, A., Gill, O. N., \& Delpech, V. C. (2014). HIV in the United Kingdom 2014 Report: data to end 2013. London: Public Health England.

Zapata, J. C. (2013, 10th May 2013). Drugs, clubs and death: what can South London's gay community do? , Gay Star News. 